\title{
Problems in the Classification of Mine Gas Grades and Classification of Coal and Gas Outburst Mine Types
}

\author{
Xianzhi Shi ${ }^{1,2}$, Yongjin Tang ${ }^{3, *}$, Xijian $\mathrm{Li}^{4}$, Jialin $\mathrm{Gao}^{2}$

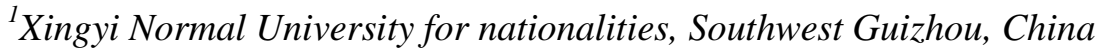 \\ ${ }^{2}$ Guizhou Yuxiang Mining Group Investment Co. Ltd. Bijie, Guizhou 550081, China \\ ${ }^{3}$ Nuodong Coal Mine, Puding County, Southwest Guizhou 562400, China \\ ${ }^{4}$ College of Mining Engineering, Guizhou University, Guizhou 550025, Guiyang, China \\ *Corresponding Author.
}

\begin{abstract}
Since the classification of coal mine gas grade, there has been a classification mode with inconsistent classification basis and progressive relationship between gas mine and coal and gas outburst mine. This paper demonstrates the irrationality of the progressive relationship between coal and gas outburst mines and gas mines from the aspects of different classification materials, inconsistent gas storage media and different disaster control measures, and determines that coal and gas outburst mines and gas mines coexist and are different types of mines. According to the actual amount of outburst coal, the outburst mines are divided into weak, medium, strong and extremely strong outburst mines by using gas content $W$, coal seam firmness coefficient $f$ and gas pressure $P$.
\end{abstract}

Key words : Gas mine; Outburst mine; Different basis; Type of outburst mine

\section{Introduction}

Before the 1980s, China's coal mines used the former Soviet Union's classification method of mine gas. After 1980, China began to classify the gas grade into three categories: low gas mine, high gas mine (both are called gas mines) and coal and gas outburst (short for outburst) mine ${ }^{[1]}$. Different scholars and literatures have put forward different opinions and opinions on the basis of classification of low-gas and high-gas mines in coal mines ${ }^{[2-5]}$. Yu Bufan suggested that the quantity of absolute gas emission in mine should be taken as the gas grade standard, and the maximum outburst intensity of the mine should be taken as the classification standard of the outburst mine ${ }^{[2]}$. Thereafter, Tu Xigen, Wang Youan and Yao Eryi put forward the classification method with the relative gas emission as the main index, the absolute gas emission as the additional index, and the annual output as the additional index ${ }^{[1]}$. Liu Yunchun and Xin Guanglong believed that when classifying the coal mine gas grade, the factors considered were not comprehensive enough and the classifications were too few. They suggested that the coal mine gas grade should be classified into four categories ${ }^{[3]}$. Bao Shangxian and Xiao Changgen studied and analyzed the gas grade identification data of 62 key coalmines in Jiangxi Province from 1980 to 2002. They suggested that the annual output of the mine should be divided into several sections and the corresponding relative gas emission value of the mine should be taken to classify the gas grade of the mine ${ }^{[4]}$. For the study of the classification of mine gas grade, the scientific researchers have been discussing the classification of high gas mine and low gas mine by the amount of mine gas emission ${ }^{[1,4]}$. The 2018 edition of "Coal Mine Gas Classification Appraisal Method" divides the gas grade according to the amount of gas emission, the form of gas emission, the actual gas dynamic phenomena and the measured outburst risk parameters. The gas grade type of coal mine is still divided into low gas mine, high gas mine and outburst mine ${ }^{[5]}$. In the course of classifying mine gas types over the years, insufficient research has been done on the basis of classification between gas mines and outburst mines, ignoring the essential differences of subject division, field control measures and disaster phenomena, and classifying mine gas grades into three progressive mine types: low gas mines, high gas mines (both are called gas mines) and outburst mines. On the basis of collecting relevant data, this paper further conducts empirical research according to coal mine gas classification, divides coal mine types according to different basis, and subdivides

ISSN: 0010-8189

(c) CONVERTER 2020 
outburst mine types according to different parameters.

\section{Analysis of the problems existing in the current classification basis}

2.1 The classification materials of outburst mine are different

The classification of gas mine is mainly based on mine gas, and the classification index is the gas emission in the mine, as is shown in Table 1. Outburst mine classification is based on outburst coal seam. The single index of outburst coal seam identification includes coal seam failure type, gas pressure, initial velocity of gas emission and firmness coefficient. The mine is divided into non outburst mine and outburst mine based on whether the coal bed is outburst or not ${ }^{[6-7]}$. Therefore, high gas mine and low gas mine may be outburst mine. The outburst mine may be low gas mine or high gas mine.

Table 1 Basis for classification of gas mines and outburst mines

\begin{tabular}{|c|c|c|}
\hline Mine types & gas mine & Outburst mine \\
\hline Classified material & Gas & Coal seam and gas \\
\hline Gas storage medium & Air & Coal seam \\
\hline $\begin{array}{c}\text { Classification } \\
\text { Indicators }\end{array}$ & $\begin{array}{c}\text { Gas emission } \\
\text { Quantity }\end{array}$ & Outburst coal seam \\
\hline
\end{tabular}

2.2. The gas occurrence medium is different by classification

The classification index of coal mine gas grade is mainly on the basis of the absolute (or relative) quantity of gas emission of the mine, taking into account the form of gas gush, ignoring the medium of gas occurrence in outburst mine and gas mine. The gas measured in gas mine exists in underground roadway and other chamber space; in outburst mine, the gas in outburst coal seam exists in coal seam pores, and the determination method of its content is different from that in low gas mine and high gas mine. The concentration of the gas in the coal seam of outburst mine can be determined only after it is analyzed and poured into the roadway from the coal seam, which can be used as the basis for dividing the low gas mine and the high gas mine.

\section{Differences in disaster management measures}

\subsection{Gas disaster and outburst disaster type analysis}

In the gas mine, when the concentration of gas in the space of the roadway reaches 5-16\%, the oxygen content is higher than $12 \%$, and the temperature reaches $650-750{ }^{\circ} \mathrm{C}$, the explosion accident can occur. In this kind of disaster, gas explosion is a process of chemical reaction. When the concentration of gas accumulation in the roadway is high, it can cause suffocation, gas combustion and other accidents.

Outburst of coal seam is a phenomenon of sudden emission of gaseous gas and solid coal in the structural coal bed with gas under the action of in-situ stress ${ }^{[7]}$. It is a geological phenomenon, mainly a physical change process. In the process of outburst and after a period of time, with the rapid increase of gas content in the air of roadway. In case of outburst, if the conditions for gas explosion are met, secondary disasters such as gas explosion may occur. For example, in the "3.31" catastrophic outburst accident of Yichuan National Coal Industry Co., Ltd., Henan Province in 2010, gas flowed from the auxiliary shaft counter current and exploded at the wellhead in case of fire ${ }^{[8]}$.

\subsection{Different disaster control measures}

3.2.1. Gas control measures

There are great differences between gas control measures and outburst preventive measures. Gas-prevention measures to "reliable ventilation, drainage standards, effective monitoring, management in place" as a comprehensive management policy, emphasizing the stability of mine ventilation, to fulfill the requirements of gas concentration in the airflow. Gas control measures mainly include gas drainage measures to prevent the gas in coal

ISSN: 0010-8189

(C) CONVERTER 2020 
seam and goaf from flowing into the roadway; monitoring measures of methane concentration in the roadway; preventive measures to prevent mine gas overrun and gas accumulation, etc. The purpose of all kinds of gas control measures is to prevent the occurrence of disasters and accidents such as personnel suffocation, gas explosion, gas combustion, etc. give rise to the excessive concentration of gas in the stope.

\subsubsection{Measures for prevention outburst}

There are many measures to prevent an outbreak. According to the regional scope of prominent prevention, it can be divided into regional integrated anti-outbreak measures and partial integrated anti-outbreak measures [6-7]. Regional integrated anti-outbreak measures and some integrated anti-outbreak measures are "four-level" integrated anti-outbreak measures. Regional outburst prevention measures mainly include mining protective seams and coal seam gas drainage. Some of the preventive measures for protruding mainly include advance drilling (including advance drilling and row drilling), hydraulic punching, loose blasting and other measures. Measures taken to prevent the protruding object is to reduce the gas content and seam, seam reducing the stress, preventing projection.

\section{Coal mine gas grade and outburst type division}

\subsection{Gas mine and outburst mine are coexisting mine types}

According to the research data ${ }^{[9,10]}$, outburst may occur in both low gas mines and high gas mines. According to the research data of Professor Jiang zebiao of Guizhou University, Yonglong coal mine and erdaoshui coal mine in Zunyi area of Guizhou Province are both low gas mines, and coal seam outburst accidents occur. The research results ${ }^{[9]}$ of Lei Shaopeng of Henan University of technology show that outburst accidents also taken place in some low gas mines and high gas mines in Zhengzhou mining area of Henan Province. According to the research results of Zhao Changwen, Yang Chaofeng and Li Zhili, Chensilou Coal Mine was identified as a low gas mine over the years before 2015. The mine mainly mined coal seam 22, with the maximum gas pressure of $0.36 \mathrm{MPa}$ and the maximum gas-bearing capacity of $6.56 \mathrm{~m}^{3} / \mathrm{t}$. The absolute quantity of gas emission is $3.52 \mathrm{~m}^{3} / \mathrm{min}$, the relative quantity of gas emission is $4.82 \mathrm{~m}^{3} / \mathrm{t}$, and the absolute quantity of gas emission is $0.65 \mathrm{~m}^{3} / \mathrm{min}$. However, an outburst trouble happened in the heading face in April 2015. On December 17, 2020, outburst taken place in Guanglong coal mine, anlong county, Guizhou Province.

Based on the analysis of relevant factors causing coal seam outburst, the material basis of outburst is the structural coal bed containing gas, and the major factors causing outburst are coal seam gas pressure (ground stress) and so ${ }^{\text {on }}{ }^{[11]}$. When the gas-bearing capacity of coal seam is low (for example, the local gas-bearing capacity of coal bed in the outburst location of Chensilou Coal Mine is $4.98 \mathrm{~m}^{3} / \mathrm{t}$ ), the outburst can occur as long as the tectonic coal and gas pressure (geostress) reach a certain value (the statistical data show that the minimum gas pressure causing the outburst occurs in Sijiazhuang coal mine of Yangmei, and the gas pressure causing the outburst is $0.4 \mathrm{MPa}{ }^{[7]}$. Therefore, outburst can occur in both low gas mine and high gas mine. Therefore, mine types can be divided into low gas coal mine and high gas coal mine according to quantity of gas emission and other indicators ${ }^{[1-5]}$, and non outburst mine and outburst mine can be divided according to whether the coal seam is outburst or not ${ }^{[6]}$, as shown in Table 2.

Table 2 classification of mine types

\begin{tabular}{|c|c|c|c|}
\hline \multicolumn{2}{|c|}{ Mine type } \\
\hline $\begin{array}{l}\text { Types of mine gas(according to gas } \\
\text { emission and other indicators) }\end{array}$ & \multicolumn{3}{|c|}{$\begin{array}{l}\text { mine outburst type } \\
\text { (according to whether the coal seam is } \\
\text { outburst or not) }\end{array}$} \\
\hline low gas mine & high gas mine & non outburst mine & outburst mine \\
\hline
\end{tabular}

\subsection{Classification of outburst mine types}

\subsubsection{Definition of outburst mine}

Coal and gas outburst, short for outburst, is the phenomenon that coal and gas suddenly gush out under the action ISSN: 0010-8189 
of in-situ stress in the structural coal bed with gas occurrence ${ }^{[11]}$. In case of coal mine outburst accident or when the coal seam dynamic phenomenon characteristics are not obvious or there is no dynamic phenomenon, according to the actual measured coal firmness coefficient $\mathrm{f}$, maximum gas pressure $\mathrm{P}$, damage type of soft layered of coal bed and initial gas emission velocity of coal $\Delta \mathrm{P}$, If all indexes reach or exceed the critical value listed in next table, it is determined as outburst coal bed, and the mine is outburst coal mine ${ }^{[6]}$.

Table 3 critical value of single index for identification of outburst coal seam

\begin{tabular}{|c|c|c|c|c|}
\hline $\begin{array}{c}\text { Judgment index of } \\
\text { outburs }\end{array}$ & $\begin{array}{c}\text { gas pressure } \\
\mathrm{P}(\mathrm{MPa})\end{array}$ & $\begin{array}{c}\text { firmness } \\
\text { coefficient } \mathrm{f}\end{array}$ & failure type & $\begin{array}{c}\text { initial velocity of } \\
\text { gas emission } \triangle \\
\mathrm{P}\end{array}$ \\
\hline $\begin{array}{c}\text { Critical value or } \\
\text { range }\end{array}$ & $\geq 0.74$ & $\leq 0.5$ & III $、 \mathrm{IV} 、 \mathrm{~V}$ & $\geq 10$ \\
\hline
\end{tabular}

\subsubsection{Classification of coal seam outburst risk}

Outburst is the result of comprehensive action of various factors, Generally speaking, there are many different factors such as coal body strength (coal seam firmness coefficient f), coal seam gas pressure P (in-situ stress), gas content of coal seam W, coal seam failure type, initial velocity of gas emission, geological structure, the thickness of coal seam, coal seam buried depth, etc; According to the definition of outburst, structural coal and gas are the material basis and internal cause of outburst; In situ stress (gas pressure) is the driving force and external cause of outburst. The practice, statistical data and experiments of mining outburst dangerous coal seam have proved that, outburst is the result of gas pressure (in-situ stress), coal strength and the gas content of coal seam, and other factors are related to or derived from these three indicators directly related to coal seam. Among these factors, the failure type of coal seam is the external manifestation of coal strength reduction, which is the result of tectonic movement. The failure degree of coal seam reflects the strength of coal body, so the failure type of coal body can be reflected by the strength value of coal body; The geological structure is the area where the in-situ stress is relatively concentrated, and the ultimate effect of the tectonic stress in the coal body is shown as the pressure gas in coal seam and coal body strength index; The initial velocity of gas emission is a manifestation of coal seam firmness coefficient.

Therefore, coal seam outburst risk can be evaluated by coal seam firmness coefficient $\mathrm{f}$, coal seam gas content $\mathrm{W}$ and coal seam gas pressure P; Combined with the quantity of coal outburst in coal seam, the outburst risk classification of outburst coal seam (i.e. the classification of outburst risk degree of outburst coal seam) is carried out, and the outburst strength of coal seam is predicted ${ }^{[11]}$, and then classify the outburst wells. Combined with coal seam outburst intensity, statistical data and production practice, according to the amount of outburst coal, using three single indexes of coal seam gas pressure, coal seam gas content and coal seam firmness coefficient, the outburst risk degree of outburst coal seam is divided into four categories: weak outburst risk, medium outburst risk, strong outburst risk and extremely strong outburst risk ${ }^{[11]}$, as shown in Table 4.

Table 4 Classification of the outburst hazard level (hazard degree)

\begin{tabular}{|c|c|c|c|c|}
\hline $\begin{array}{c}\text { Types of coal and } \\
\text { gas outburst } \\
\begin{array}{c}\text { Mine } \\
\text { classification index }\end{array}\end{array}$ & $\begin{array}{c}\text { weak } \\
\text { outburst } \\
\text { mine }\end{array}$ & $\begin{array}{c}\text { medium } \\
\text { outburst } \\
\text { mine }\end{array}$ & $\begin{array}{c}\text { strong } \\
\text { outburst } \\
\text { mine }\end{array}$ & $\begin{array}{c}\text { very } \\
\text { strong } \\
\text { outburst } \\
\text { mine }\end{array}$ \\
\hline $\begin{array}{c}\text { Amount of outburst coal } \\
\text { (rock) }(t)\end{array}$ & $<100$ & $100 \sim 500$ & $500 \sim 1000$ & $\geq 1000$ \\
\hline gas pressure $\mathrm{P}(\mathrm{MPa})$ & $0.4 \sim 0.5$ & $0.5 \sim 1.5$ & $1.5 \sim 2.0$ & $\geq 2.0$ \\
\hline firmness coefficient $\mathrm{f}$ & $>1.2$ & $1.2 \sim 0.5$ & $0.5 \sim 0.3$ & $\leq 0.3$ \\
\hline Gas content $W\left(\mathrm{~m}^{3} / t\right)$ & $<5$ & $5 \sim 12$ & $12 \sim 16$ & $\geq 16$ \\
\hline
\end{tabular}

\subsubsection{Classification of outburst mine types}

ISSN: 0010-8189

(C) CONVERTER 2020

www.converter-magazine.info 
According to the actual outburst coal quantity, the outburst mines are divided into weak outburst mines, medium outburst mines, strong (serious) and extremely strong (extremely serious) outburst mines ${ }^{[11-13]}$, as shown in Table 5.

Table 5 Classification of outburst mine types according to the amount of outburst coal

\begin{tabular}{|c|c|c|c|c|}
\hline $\begin{array}{c}\text { Mine } \\
\text { Types of coal and } \\
\text { gas outburst } \\
\text { classification index }\end{array}$ & $\begin{array}{c}\text { weak } \\
\text { outburst } \\
\text { mine }\end{array}$ & $\begin{array}{c}\text { medium } \\
\text { outburst } \\
\text { mine }\end{array}$ & $\begin{array}{c}\text { strong } \\
\text { outburst } \\
\text { mine }\end{array}$ & $\begin{array}{c}\text { very strong } \\
\text { outburst mine }\end{array}$ \\
\hline Amount of outburst coal (rock) $(t)$ & $<100$ & $100 \sim 500$ & $500 \sim 1000$ & $\geq 1000$ \\
\hline
\end{tabular}

According to the firmness coefficient of coal seam, the gas content of coal seam, and the gas pressure of coal seam index evaluation, combined with the data in Table 2 and table 4, outburst mines can be divided into four categories, namely weak outburst mine, medium outburst mine, strong (serious) outburst mine and very strong (very serious) outburst mine, as shown in Table 6.

Table 6 Classification of outburst mine types

\begin{tabular}{|c|c|c|c|c|}
\hline $\begin{array}{c}\text { Types of coal and } \\
\text { gas outburst } \\
\text { classification index }\end{array}$ & $\begin{array}{c}\text { weak } \\
\text { outburst } \\
\text { mine }\end{array}$ & $\begin{array}{c}\text { medium } \\
\text { outburst } \\
\text { mine }\end{array}$ & $\begin{array}{c}\text { strong } \\
\text { outburst } \\
\text { mine }\end{array}$ & $\begin{array}{c}\text { very } \\
\text { strong } \\
\text { outburst } \\
\text { mine }\end{array}$ \\
\hline gas pressure $\mathrm{P}(\mathrm{MPa})$ & $0.4 \sim 0.5$ & $0.5 \sim 1.5$ & $1.5 \sim 2.0$ & $\geq 2.0$ \\
\hline firmness coefficient $\mathrm{f}$ & $>1.2$ & $1.2 \sim 0.5$ & $0.5 \sim 0.3$ & $\leq 0.3$ \\
\hline Gas content $W\left(\mathrm{~m}^{3} / \mathrm{t}\right)$ & $<5$ & $5 \sim 12$ & $12 \sim 16$ & $\geq 16$ \\
\hline
\end{tabular}

\section{Conclusion}

(1) The current classification of coal mine gas in China ignores the differences of gas occurrence medium, classification basis and disaster control measures between gas mine and outburst mine.

(2) Gas mines and outburst mines are not progressive, but juxtaposed mine types. According to the index of mine gas emission, the mine is divided into low gas mine and high gas mine; According to the coal seam outburst or not, the mine is divided into outburst mine and non outburst mine. According to the coal seam gas pressure, firmness coefficient and coal seam gas content index, the outburst mine can be divided into weak, medium, strong and extremely strong outburst mine.

(3) The classification of gas mines and outburst mines is conducive to distinguish the prevention and control of outburst from the gas control in "one ventilation and three prevention" in coal mine production practice, which can further promote the development of gas geology in Colleges and universities, improve the professional technology level of outburst prevention in coal mines, and reduce the occurrence of outburst accidents.

\section{References}

[1] X. G. Tu, Wang Youan, Yao Eryi. Suggestions on classification of mine gas. Coal mine safety. 22-26, 1998(9).

[2] B. F. Yu, Discussion on classification of mine gas grade. Coal engineer. 15-23, 1992(1).

[3] Y. C. Liu, G. L. Xin, Discussion on classification of mine gas grade. Coal mine safety, 1999(8), 34-35.

[4] S. X. Bao, C. G. Xiao, Suggestions on defining gas grade by using mine gas emission. Jiangxi Coal Science and technology, 2003(3), 1-5.

[5] State Administration of coal mine safety, state energy administration. Coal mine gas grade identification method. Beijing. Coal Industry Press, 2018.

ISSN: 0010-8189

(C) CONVERTER 2020 
[6] State Administration of coal mine safety. Detailed rules for prevention and control of coal and gas outburst. Beijing: Coal Industry Press, 2019.

[7] H. S. Wang, X. Z. Shi, Theory and technology for prevention and control of coal and gas outburst. Xuzhou: China University of mining and Technology Press, 2014.

[8] Z. X. Li, T. M. Wang, S. Y. Wang, et al. Simulation study of ventilation system disaster evolution in coal and gas outburst mine. Journal of China Coal Society. 42(4), 929-934, 2017.

[9] S. P. Lei, Study on characteristics of coal and gas outburst in low gas coal mine. Jiaozuo, Henan: Henan University of technology, 2017.

[10] Liu Mingju, Zhao Wenwu, Wei Jianping, et al. Statistical analysis and preventive measures of coal mine gas outburst accidents in China in 2006. Coal technology, 27 (11). 79-81, 2008.

[11] X. Z. Shi, D. Z. Song, Z. W. Qian, Classification of coal seam outburst hazards and evaluation of the importance of influencing factors. Open Geosci, 2017(9), 295-301.

[12] Y. A. Wang, Discussion on classification of risk degree of coal and gas outburst mine. Coal mine safety, 1991(9), 38-43.

[13] W. Nie, Y. Liu, C. J. Li, and J. Xu.A Gas Monitoring and Control System in a Coal and Gas Outburst Laboratory. Journal of Sensors, 46(3).727-737, 2014. 